\title{
Deficiencias en la enseñanza de las matemáticas en el nivel primario de la educación básica general de Panamá
}

Pág. 207- 223

*José Ángel

González C.

***Yoshiko Saito

*Universidad de Panamá

Centro Regional Universitario de Veraguas

Departamento de Matemáticas

$\underline{\text { angelojose3021@gmail }}$ .com

***Voluntaria de la Agencia de Cooperación Internacional del Japón -JICA- en la Escuela Normal Juan Demóstenes Arosemena.

Maestra de enseñanza primaria

aozorays@gmail.com

Fecha de

Entrega: noviembre de 2019.

Fecha de Aceptación: diciembre de 2019.

\section{Resumen}

Este artículo es el resultado de una investigación cuyo objetivo fue analizar los contenidos curriculares de los programas de matemáticas de primero a sexto grado de educación primaria de la educación básica general, en base a resultados obtenidos en pruebas internacionales realizadas en el año 2017. El alcance de esta investigación incluyó estudiantes del nivel superior y bachilleres de la Escuela Normal Demóstenes Arosemena, estudiantes de primer ingreso de la Universidad de Panamá, estudiantes de séptimo grado y maestros en ejercicio; todos de la Provincia de Veraguas. La metodología fue de tipo descriptiva-cualitativa, realizando un estudio comparativo entre nuestro currículo de matemática y el de cinco países latinoamericanos incluyendo Japón, así como pruebas realizadas a estudiantes y maestros. Los resultados evidenciaron un bajo dominio en temas fundamentales del currículo de matemática del nivel primario. El estudio permitió también constatar que los currículos de Chile, México, Costa Rica, Guatemala, Honduras; países que salieron mejor que el nuestro en la prueba TERCEpresentan una unidad y coherencia curricular; mientras que Panamá posee un currículum de matemáticas con una distribución diferente, dato este considerado de suma importancia en esta investigación.

\section{Palabras clave}

Currículo, matemática, enseñanza, aprendizaje, metodología. 


\title{
Deficiencies in Mathematics Teaching at the Primary Level of
} General Basic Education in Panama

\begin{abstract}
This article is the result of a research aimed at analyzing the curricula content of math programs from first to sixth grade of primary education in general basic education, based on the results obtained in international tests administered in 2017. The scope of this research included senior and high school students of the Demostenes Arosemena Normal School, Universidad de Panama freshmen, seventh graders and full-time teachers; all of them from the Province of Veraguas. We used a descriptive-qualitative methodology, conducting a comparative study between our math curriculum and that of five Latin American countries and Japan, in addition to tests administered to students and teachers. The results evidenced a low mastery level in fundamental topics related to the primary level math curriculum. The study also showed that the curricula of Chile, Mexico, Costa Rica, Guatemala, Honduras; countries that got a better performance than ours in the TERCE test - have a curricular unity and coherence; while Panama has a math curriculum with a different distribution, this fact is considered of utmost important in this research.
\end{abstract}

\section{Keywords}

Curriculum, mathematics, teaching, learning, methodology. 


\section{Introducción}

El sistema educativo panameño ofrece el acceso gratuito, universal y obligatorio para los niños y jóvenes en edad de asistir a la educación básica general; según el último informe del Ministerio de Educación se ha ampliado la capacidad de las aulas escolares; se le ha dado acceso a la tecnología, a niños en educación preescolar y aunque está presente en muchas escuelas, el acceso todavía es reducido y aún existen limitaciones en cuanto a infraestructuras escolares.

En cuanto a la educación primaria, si bien existe una alta cobertura, todavía se registra una alta deserción escolar y la brecha entre los niveles de escuelas urbanas y escuelas rurales o de áreas indígenas, es bastante evidente (MEDUCA, 2017, pp. 25-26).

Sin embargo, una de las deficiencias más notorias en esta descripción breve de nuestro sistema educativo, es el alto índice de deficiencias en la asignatura de matemática, la cual se refleja en todos los grados de escolaridad. Esta situación tiene una incidencia directa en los resultados de las diferentes pruebas de medición tanto nacionales como regionales en las cuales ha participado nuestro país, al igual que en las pruebas de admisión para el ingreso en las instituciones de educación superior.

La República de Panamá participó de la prueba TERCE en el año 2013 como parte del proceso de evaluación de la calidad educativa a nivel regional promovido por la Oficina Regional de Educación para América Latina y el caribe/UNESCO sede Santiago de Chile.

En esta prueba, desarrollada según la perspectiva curricular de los países participantes, se obtuvieron resultados de los logros en estudiantes de tercer y sexto grado en las áreas de lectura, 
matemáticas, ciencias naturales y escritura. Para los efectos de esta investigación partimos de los resultados según las puntuaciones promedio en las pruebas de matemática para tercero y sexto grado.

En este caso los resultados fueron los siguientes:

- Prueba de matemática para estudiantes de tercer grado. Puntaje obtenido 664 puntos, ubicándose por debajo de México, Honduras, Guatemala, C. Rica, Colombia, Chile, Brasil y Argentina.

- Prueba de matemática para estudiantes de sexto grado. Puntaje obtenido 644 puntos, ubicándose por debajo de México, Honduras, Guatemala, C. Rica, Colombia, Chile, Brasil y Argentina (OREALC/UNESCO, 2015).

Estos resultados evidenciaron realidades sumamente preocupantes para nuestro sistema educativo ya que mostraron la existencia de graves fallas en todo el proceso de enseñanza aprendizaje, desde el diseño mismo de los programas de estudio, hasta los métodos que utiliza el maestro para desarrollar los contenidos.

Sin embargo, tampoco parece haber un sistema de realimentación que provea al docente de información útil basada en los resultados de estas pruebas (BID/Diálogo Interamericano y Unido por la Educación, 2018, p. 21). 
Este escenario educativo, producto de los resultados de la prueba TERCE, constituye una de las principales motivaciones que nos indujo a desarrollar una investigación académica con el fin de conocer las posibles causas por las cuales nuestros estudiantes no logran alcanzar los niveles esperados en este tipo de pruebas.

Es por esto que en el año 2017 realizamos en calidad de investigador, junto a la profesora Yoshiko Saito, voluntaria de la JICA (Agencia de Cooperación Internacional de Japón) un estudio cualitativo de la realidad educativa de primero a sexto grado, en cuanto a la enseñanza de la matemática.

Este estudio lo hicimos tomando en cuenta dos fases importantes de la investigación, en primer lugar, se analizaron los programas de estudio de la asignatura matemática y el cartel de alcance y secuencia de primero a sexto grado de la Educación Básica General en Panamá.

El propósito en esta primea fase fue determinar la secuencia lógica -horizontal y vertical- de los contenidos de los programas, si los contenidos de cada grado correspondían a los niveles de madurez psicológica del niño.

En esta fase también se hizo un análisis comparativo de los contenidos conceptuales en el área de aritmética de nuestros programas en relación con los programas de cinco países latinoamericanos que obtuvieron mejores resultados que el nuestro en la prueba TERCE: Honduras, Guatemala, Costa Rica, México, Chile. Es importante destacar que gracias a la participación de la profesora Yoshiko Saíto en la investigación, se tomó también como referencia en este estudio, el programa de Japón en estos grados de escolaridad. 
En segundo lugar, como parte de este proceso de investigación también se hizo un estudio observacional para determinar el enfoque metodológico empleado por los maestros en la enseñanza de la matemática, el uso de materiales didácticos pertinentes y la planificación del maestro para desarrollar el programa. En este punto fue de gran importancia para los investigadores conocer la dosificación e integración de las áreas, el dominio de los contenidos y, si la carga horaria semanal que dispone el maestro durante el año, era suficiente para desarrollar el programa y evaluar el nivel de logro de los alumnos en cuanto a la comprensión de los conceptos, la resolución de problemas y el dominio de operaciones básicas.

\section{Metodología}

La metodología utilizada en esta investigación, fue de tipo cuantitativo descriptiva, utilizando para ello varias metodologías e instrumentos.

a. Pruebas sobre operaciones básicas como suma, resta, multiplicación y división y cálculos aritméticos que involucran la aplicación de estas operaciones. Esta prueba tiene un valor de 100 puntos y los contenidos considerados, corresponden al programa de primero a tercer grado de la enseñanza primaria de Panamá. La muestra incluye estudiantes de los niveles superior, medio, pre media y primaria, tal y como se detalla a continuación:

- 100 estudiantes de segundo año del Instituto Pedagógico Superior Juan Demóstenes Arosemena.

- 30 estudiantes de primer ingreso del Centro Regional Universitario de Veraguas (estudiantes, recién egresados de media) 
- 200 estudiantes del bachiller pedagógico de la Escuela Normal Juan Demóstenes Arosemena. (Nivel media)

- 150 estudiantes del séptimo grado de la Escuela Normal Juan Demóstenes Arosemena. (Nivel pre media).

- 60 estudiantes de una escuela primaria de la ciudad de Santiago, Provincia de Veraguas (nivel primario).

La razón por la cual se trabajó con esta muestra radica en el hecho que la Escuela Normal Juan Demóstenes Arosemena y el Instituto Pedagógico Superior "Juan Demóstenes Arosemena", son los únicos formadores de maestros en el país. En cuanto al grupo (universitario) de primer ingreso, éste se eligió para determinar las competencias y habilidades que tienen los estudiantes que provienen del segundo nivel de enseñanza.

b. Encuesta dirigida a maestros para determinar su percepción acerca de los Programas de estudio de matemática y los materiales didácticos utilizados para la enseñanza. Como muestra y de forma aleatoria, se tomó el universo de los maestros de una Escuela Primaria, ubicada en la ciudad de Santiago, Provincia de Veraguas la cual cuenta con 30 docentes y 656 estudiantes. La encuesta consta de 15 preguntas.

c. Observación en el Aula: se utilizó la lista de cotejo, con 20 criterios, orientados a verificar la metodología utilizada por los maestros en la enseñanza del cálculo mental y la resolución de problemas; así como el uso que se le da a los textos y materiales didácticos para la enseñanza de la matemática. Esta observación se realizó en 5 aulas por un espacio de 5 meses. 
d. Análisis descriptivo de los Programas de estudio de matemática de primero a sexto grado y el cartel de alcance y secuencia correspondiente.

e. Estudio comparativo de los contenidos de los programas de matemática de primero a sexto grado de Panamá con los programas de cinco países latinoamericanos y Japón.

\section{Resultados de la investigación.}

a. Identificación de situaciones críticas en el área de la matemática.

De la aplicación de las pruebas realizadas en los diferentes niveles, con base a 100\%, se obtuvo los siguientes resultados:

\section{Tabla 1}

Resultados de las pruebas en los diferentes niveles

Nivel

Nivel Superior (Instituto Pedagógico Superior)

Resultados

Nivel Media (bachiller pedagógico -Escuela Normal Juan Demóstenes $71 \%$

Arosemena)

Estudiantes de primer ingreso (universitario) - Universidad de Panamá $75 \%$

Nivel Pre media- Escuela Normal Juan Demóstenes Arosemena $\quad 56 \%$

Nivel primario

$46 \%$

Fuente: Elaboración propia de acuerdo a resultados de las pruebas.

Partiendo del hecho que la prueba consideró contenidos aritméticos de los programas de primero a tercer grado de enseñanza primaria, consideramos que estos resultados demuestran que en general todos los sujetos estudiados tienen dificultades para realzar cálculos aritméticos básicos, que debieran ser aprendidos en los primeros grados de escolaridad. 


\section{b. Resultados de las encuestas aplicadas}

- En cuanto a la parte técnica curricular los encuestados manifestaron exceso de contenidos en los programas, lo cual trae como consecuencia directa que el maestro no tiene tiempo suficiente para desarrollar las áreas que establecen los DFA (Deberes Fundamentales de Aprendizaje o DBA según otros países).

- Un 75\% manifestó que el área del programa más fácil para enseñar, es el “área de número", sin embargo, en la prueba aplicada se evidencia dificultades en esta área del programa.

- Un $69 \%$ considera que el sistema educativo no funciona bien, en cuanto a que no ha podido responder ni resolver, muchos de los problemas que el proceso de transformación curricular (iniciado en 2015) ha dejado en el camino.

- -En cuanto al uso de materiales didácticos, los encuestados indican que los utilizan, pero que les gustaría recibir capacitaciones sobre su mejor uso y diseño, para utilizarlos en sus clases.

\section{c. Observación en el aula con lista de cotejo:}

De la observación en el aula con lista de cotejo se evidencia que:

- Los estudiantes observados presentan claras deficiencia en la realización de cálculo mental sobre operaciones elementales tales como multiplicación, suma, resta y división. Esto se evidencia en la manera en las actividades de seguimiento y evaluación que realizan los maestros diariamente.

- Los maestros no disponen de suficientes recursos didácticos y materiales de enseñanza.

- -El enfoque utilizado no parte de lo concreto para llegar a lo pictórico y abstracto. 
- Los maestros demuestran deficiencias en las habilidades básicas para la resolución de problemas matemáticos.

- Los textos que se utilizan, no facilitan estrategias pedagógicas que conduzcan al niño al desarrollo de habilidades para la resolución de problemas y tampoco ayudan al maestro a integrar las diferentes áreas del programa; más bien su objetivo principal es el desarrollo de los contenidos.

- La enseñanza es mecánica y abstracta, sin aplicación en situaciones de su entorno. Esta situación resta aprecio y atención del estudiante, no encontrando sentido de lo que aprende”.

- Los maestros, atentos a cumplir con la gran cantidad de contenidos que indica el Programa, priorizan en el volumen de temas, en detrimento de la calidad y profundidad de los aprendizajes. Esta presión, que se expresa en "falta de tiempo", es un obstáculo que conduce a una enseñanza apresurada y superficial".

- La metodología empleada por los maestros en la enseñanza de la matemática es todavía tradicional; se prefiere la clase teórica centrada en el tablero, en vez de un aprendizaje basado en actividades didácticas, la resolución de problemas, el uso de recursos didácticos para gestionar un aprendizaje que vaya de lo concreto a lo abstracto, de lo conocido a lo desconocido y de lo fácil a lo difícil.

\section{d. El análisis descriptivo de los Programas estudio de matemática de primero a sexto} grado y el cartel de alcance y secuencia correspondiente, nos reveló lo siguiente:

- Contenidos curriculares que no corresponden al desarrollo cognitivo del niño.

- Existencia de repetición innecesaria de contenidos, lo cual indica que no existe una secuencia didáctica progresiva en cuanto a los contenidos. 
- El mismo programa no permite al estudiante desarrollar habilidades y competencias matemáticas para la resolución de problemas.

- La cantidad de horas en el plan de estudios no es suficiente para desarrollar el programa ya que el mismo es extenso.

\section{e. Estudio comparativo.}

Se consideró revisar nuestros Programas, en relación a los de otros países de la región (México, Guatemala, Honduras, Costa Rica, Chile) y Japón. Esto se realizó para el área de Aritmética, la cual incluye aspectos de suma, resta, multiplicación, división, fracción, decimal, potenciación, radicación; considerando que ésta área es básica para el desarrollo de otras competencias matemáticas más complejas.

Los resultados se muestran en la Tabla 2.

\section{Tabla 2}




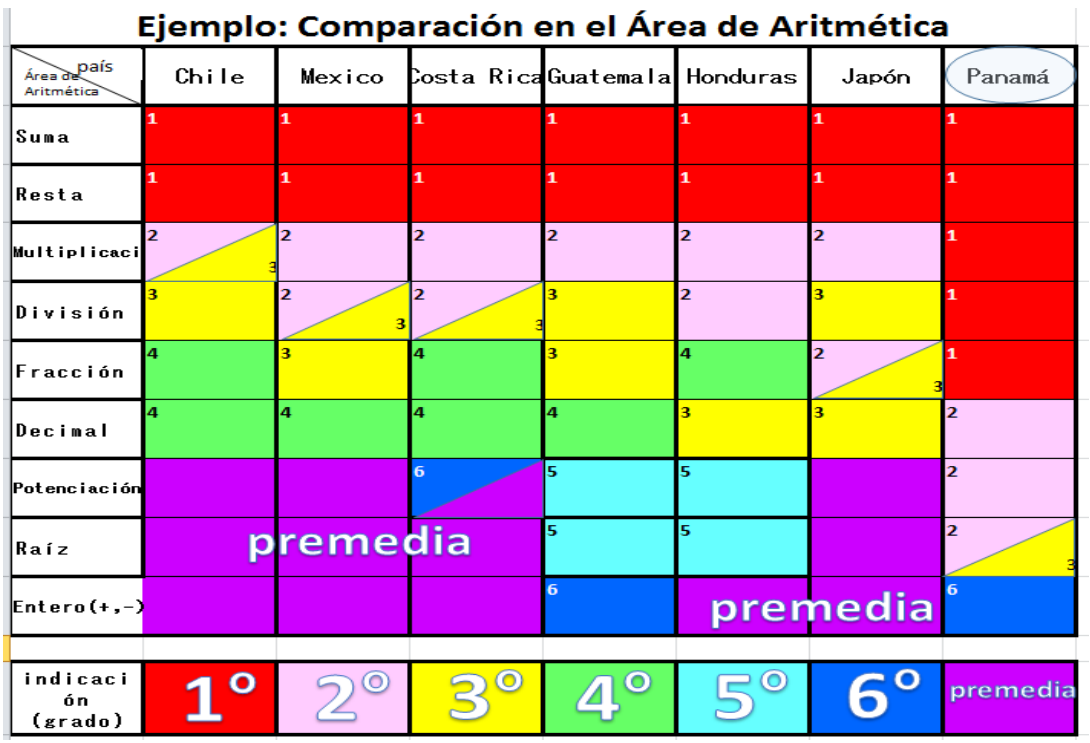

Fuente: Programas de estudio y textos de Chile, México, C.

Rica, Guatemala, Honduras, Japón y Panamá.

En el mismo, podemos observar que: La suma y resta de números naturales se enseña en primer grado en todos los países, incluyendo Panamá, sin embargo, el punto diferencial que nos llama la atención y sobre el cual sustentamos parte de nuestro análisis es que, mientras en Chile, México, Costa Rica, Guatemala, Honduras y Japón existe una distribución de las áreas de la aritmética (suma, resta, multiplicación, fracción, decimal, potenciación, radicación, número entero), en Panamá la distribución es diferente.

Así vemos que mientras en los demás países la suma y resta se da en el primer grado para luego pasar a la multiplicación en segundo grado y la división en segundo y tercero; en Panamá nuestro currículo aborda las cuatro operaciones básicas y el concepto de fracción y potenciación en segundo grado.

Es decir, existe un fraccionamiento altamente concentrado en cuanto a la enseñanza de la suma, 
resta, multiplicación, y división en un solo nivel, lo cual tiene como resultado que el aprendizaje de estas áreas quede a medias.

Si observamos nuevamente el cuadro en cuestión vemos que en estos países el tema de la suma y resta se enseña durante todo el primer grado procurando que los conceptos se adquieran plenamente llevando al estudiante a niveles de razonamiento y desarrollando el pensamiento lógico, de tal forma que en el momento en que el estudiante pasa al siguiente nivel, éste cuenta con las competencias necesarias para enfrentar la multiplicación y otras operaciones, la cual requiere de dominio pleno de la suma y la resta.

Incluso resulta sorprendente que temas como: potenciación y radicación, se enseñe en segundo grado en el sistema escolar de Panamá, cuando las mismas deberían enseñarse en Pre-media. Analizar el concepto matemático de potencia significa conocer dominios que involucran el sistema de signos de la actividad matemática que el niño va adquiriendo de acuerdo a su madurez mental.

Por la antes expuesto el concepto de potencia y radicación requiere del dominio de estas estructuras mentales por lo que es necesario darle la oportunidad al niño de adquirir ciertas destrezas para el dominio de este tema.

\section{Conclusiones}

La enseñanza de las matemáticas constituye uno de los pilares básicos, fundamentales y prioritarios de cualquier sistema educativo. Los resultados de este estudio, permiten concluir que existen graves deficiencias en la enseñanza de las matemáticas que ameritan ser atendidas en 
el corto plazo. Así, por ejemplo, en cuanto a la formación del maestro en el área de matemáticas, la prueba aplicada a estudiantes del Instituto Pedagógico Superior Juan Demóstenes Arosemena y maestros en ejercicio; deja ver carencias en la formación, que limitan el ejercicio de la enseñanza de las matemáticas. Es evidente, a la luz de los resultados, que existe un bajo dominio de temas fundamentales como operaciones básicas, geometría, medidas, las cuales complementan el currículo, ya que estas áreas se deben correlacionar en el proceso de enseñanza aprendizaje.

Igualmente existen grados de dificultad por parte de maestros para la enseñanza de áreas como la aritmética, (perímetro, área, porcentaje, entre otros) y un desconocimiento de la profundidad de los conceptos básicos matemáticos, que originan conceptos más complejos.

El tema del concepto matemático es fundamental y un desconocimiento o poco dominio de éste tiene una repercusión directa en el manejo de la propia didáctica de la matemática. Precisamente la investigación evidenció el poco dominio, por parte de los maestros, del uso de metodologías y técnicas didácticas para la enseñanza, así como la falta de contextualización de los aprendizajes. Por otro lado, la observación de clase evidencia que el maestro se enfoca más en cubrir los contenidos del Programa que en asegurar la calidad de los aprendizajes. Esta presión que parecen ejercer los Programas, se agrava con las temáticas abordadas y la falta de dosificación por grado, aspecto que evidentemente es subsanado en otros países.

Entonces, ¿qué perspectivas apuntan los resultados de esta investigación? la respuesta es contundente: si continuamos con la misma práctica educativa, sustentada en el mismo formato curricular que tenemos en la actualidad, los resultados -lastimosamente para nuestro país- 
seguirán siendo los mismos. Por lo tanto, concluimos que es urgente la revisión de todos los componentes curriculares de la asignatura de matemática para el nivel primario y que es necesario repensar el currículum de formación de maestros en materia de didáctica para la enseñanza de las matemáticas.

Como corolario de lo anterior tenemos que en el año 2018 Panamá realizó la prueba CRECER y los resultados de ésta, fueron muy similares a los de la prueba TERCE. Estos resultados de la prueba CRECER, ya se habían previsto a la luz de esta investigación, de aquí la urgencia de transformar el currículo de matemática, la creación de nuevos textos basados en un enfoque concreto-pictórico-abstracto y revisar los programas de formación del maestro de enseñanza primaria.

\section{Referencias}

BID/Diálogo Interamericano y Unido por la Educación. (2018). Panamá. El estado de las políticas públicas docentes. Recuperado el 2919, de https://www.thedialogue.org/wpcontent/uploads/2018/08/Panam\%C3\%A1_FINAL.pdf/.

Gobierno de Chile. Ministerio de educación. (2019). Unidad de Currículum y Evaluación. Recuperado el 2020, de Curriculum Nacional: Matemática: https://www.curriculumnacional.cl/614/w3-propertyvalue-49395.html/.

Gobierno de la República de Guatemala. Ministerio de Educación. (2011). Dirección General de Gestión de Calidad Educativa. Recuperado el 2020, de Libros de Matemática. Ciclo Básico: https://www.mineduc.gob.gt/DIGECADE/Guatematica.asp/. 
Gobierno de México. Ministerio de Educación. (2020). Mapa curricular. Recuperado el 2020, de https://www.planyprogramasdestudio.sep.gob.mx/index-mapa-curricular.html/.

Gobierno de Panamá. Ministerio de Educación. (2019). Educa Panamá. Mi portal educativo. Recuperado el 2020, de http://www.educapanama.edu.pa/?q=planes-y-programas-deestudios.

MEDUCA. (2017). Compromiso nacional por la educación. Meduca PNDU. Recuperado el 2019,

https://www.undp.org/content/dam/panama/docs/Documentos_2018/Compromiso-NalEducacion-WEB.pdf/.

Ministerio de Educación. (2019). Aprendizajes Fundamentales de Matemática para los grados 1 $\begin{array}{llllll}a & 6 & \text { el } & \text { Recuperado }\end{array}$ http://www.meduca.gob.pa/sites/default/files/prensa/images/Octubre\%202017/Panama matem\%C3\%A1ticas_31\%20de\%20mayo.pdf/.

Ministerio de Educación. Gob. de la República de Guatemala. (2020). Dirección General de Gestión de Calidad Educativa. Recuperado el 2020, de http://www.mineduc.gob.gt/DIGECADE/.

OREALC/UNESCO. (Junio de 2015). Logros de aprendizaje. Prueba TERCE/Tercer estudio regional comparativo y explicativo. Recuperado el 2019, de Logros de aprendizaje/Laboratorio Latinoamericano de evaluación de la calidad de la educación.: https://unesdoc.unesco.org/ark:/48223/pf0000243532/PDF/243532spa.pdf.multi/.

República de Costa Rica. Ministerio de Educación Pública. (2019). Reforma curricular en ética, estética y ciudadanía. Recuperado el 2020, de Programa de matemáticas. I y II Ciclo de la Educación Primaria. III Ciclo de Educación G.Básica: https://www.mep.go.cr/sites/default/files/programadeestudio/programas/matematica.pdf/. 
República de Honduras. Secretaría de educación. (2019). Diseño Curricular Nacional para la Educación Básica. Recuperado el 2020, de https://www.se.gob.hn/media/files/basica/DCNEB_primer_ciclo.pdf/. 